\title{
A TEST FOR COMMUTATORS
}

\section{by HANS LIEBECK}

(Received 31 October, 1974)

1. Introduction. In the course of a study of commutator subgroups I. D. Macdonald [1] presented the free nilpotent group $G_{4}$ of class 2 on 4 generators as an example of a nilpotent group whose commutator subgroup has elements that are not commutators. To demonstrate this he proceeded as follows: let $G_{4}=\left\langle a_{1}, a_{2}, a_{3}, a_{4}\right\rangle$ and put $c_{i j}=\left[a_{i}, a_{j}\right]$ for $1 \leqq i<j \leqq 4$. Then the relations in $G_{4}$ are $\left[c_{i j}, a_{k}\right]=1$ for $1 \leqq i<j \leqq 4$ and $1 \leqq k \leqq 4$, and their consequences. Macdonald observed that an arbitrary commutator may be written as

which simplifies to

$$
\left[a_{1}^{\alpha_{1}} a_{2}^{\alpha_{2}} a_{3}^{\alpha_{3}} a_{4}^{\alpha_{4}}, \quad a_{1}^{\beta_{1}} a_{2}^{\beta_{3}} a_{3}^{\beta_{3}} a_{4}^{\beta_{4}}\right],
$$

$$
\prod_{1 \leqq i<j \leqq 4} c_{i j}^{\delta_{i j}},
$$

where $\delta_{i j}=\alpha_{i} \beta_{j}-\alpha_{j} \beta_{i}$. The indices $\delta_{i j}$ satisfy the relation

$$
\delta_{12} \delta_{34}-\delta_{13} \delta_{24}+\delta_{14} \delta_{23}=0 .
$$

It follows that the element $c_{13} c_{24}$ in $G_{4}^{\prime}$ (for which $\delta_{12}=\delta_{14}=\delta_{23}=\delta_{34}=0$ and $\delta_{13}=\delta_{24}$ $=1$ ) is not a commutator.

This note was prompted by the discovery that relation (1) is not only necessary but also sufficient for the element $\Pi c_{i j}^{\delta_{i j}}$ to be a commutator in the group $G_{4}$. We shall prove a generalization to finitely generated nilpotent groups of class 2 . As an application we prove that if the commutator subgroup $G^{\prime}$ of a group $G$ is central in $G$ and can be generated by one or two elements, then every element of $G^{\prime}$ is a commutator. The case of cyclic commutator subgroups was considered by Macdonald [2] by a different method. (He proved that $G^{\prime}$ is generated by a commutator, but it is an immediate consequence that $G^{\prime}$ consists entirely of commutators.)

2. The main theorem. Throughout this section let $G$ denote a nilpotent group of class 2 that is generated by elements $a_{1}, \ldots, a_{n}$, which need not be independent. Put $c_{i j}=\left[a_{i}, a_{j}\right]$ for $1 \leqq i<j \leqq n$. Then $c_{j i}=c_{i j}^{-1}$. An element $c$ of the commutator subgroup $G^{\prime}$ is expressible in the form

$$
c=\prod_{1 \leqq i<j \leqq n} c_{i j}^{\delta_{1 j}}
$$

It is convenient to set $\delta_{j i}=-\delta_{i j}$ (and in particular $\delta_{i i}=0$ ) for all $1 \leqq i, j \leqq n$. We associate with the expression (2) for the element $c$ the $n \times n$ skew-symmetric matrix $\Delta=\left(\delta_{i j}\right)$. Put

$$
\Delta_{q r s t}=\delta_{q r} \delta_{s t}-\delta_{q s} \delta_{r t}+\delta_{q t} \delta_{r s}
$$

whenever $1 \leqq q, r, s, t \leqq n$. The following rules are easily verified:

(a) If $\tau$ is a transposition interchanging two of the symbols $q, r, s, t$, then

$$
\Delta_{q t r i s t t}=-\Delta_{q r s t}
$$


(b) If two of $q, r, s, t$ are equal then $\Delta_{q r s t}=0$.

We have the following result.

THEOREM 1. The element $c$ of $G^{\prime}$ as given by (2) is a commutator if the associated matrix $\Delta$ satisfies

$$
\Delta_{q r s t}=0 \text { for all } 1 \leqq q, r, s, t \leqq n .
$$

REMARK. In view of properties (a) and (b), conditions (4) are equivalent to the conditions $\Delta_{\text {qrst }}=0$ whenever $1 \leqq q<r<s<t \leqq n$. These conditions hold trivially when $n \leqq 3$, so a special case of Theorem 1 is that $C^{\prime}$ consists entirely of commutators whenever $G$ can be generated by not more than three elements.

Before proving Theorem 1 we shall establish the following lemma concerning the effect that certain changes of generating set of $G$ have on the representation of $c$ as a product of commutators. This will allow us to prove the existence of a presentation relative to which $c$ is clearly displayed as a commutator.

Lemma 1. Consider the following types of "elementary" changes of generating sets of G:

Type I(Transposition): $a_{i}^{*}=a_{i r}$ for all $i$, where $\tau$ is a transposition $(k l), 1 \leqq k, l \leqq n$.

Type II (Inversion): $a_{k}^{*}=a_{k}^{-1}, a_{i}^{*}=a_{i}$ for all $i \neq k$.

Type III (Combination): $a_{k}^{*}=a_{k} a_{l}^{\alpha}(k \neq l, \alpha$ an integer $), a_{i}^{*}=a_{i}$ for all $i \neq k$.

Put $c_{i j}^{*}=\left[a_{i}^{*}, a_{j}^{*}\right]$. Let the element $c$ of $G^{\prime}$ be expressible as

$$
c=\prod_{1 \leqq i<j \leqq n} c_{i j}^{\delta_{j} j}
$$

relative to the generating set $\left\{c_{i j}, 1 \leqq i<j \leqq n\right\}$ of $G^{\prime}$. Then there exists an expression for $c$ of the form

$$
c=\prod_{1 \leqq i<j \leqq n} c_{i j}^{* \delta_{i j}},
$$

where the entries of the corresponding skew-symmetric matrices $\Delta=\left(\delta_{i j}\right)$ and $\Delta^{*}=\left(\delta_{i j}^{*}\right)$ are related as follows:

Type I:

$$
\begin{gathered}
\delta_{i j}^{*}=\delta_{i \mathrm{r} j \mathrm{r}} ; \text { in particular } \delta_{k l}^{*}=\delta_{l k}=-\delta_{k l} . \\
\Delta_{q r s t}^{*}=\Delta_{q \mathrm{trrstr} .}
\end{gathered}
$$

Type II:

$$
\delta_{i k}^{*}=-\delta_{i k} \text { for all } i ; \delta_{i j}^{*}=\delta_{i j} \text { otherwise. }
$$

$$
\Delta_{\text {qrst }}^{*}=(-1)^{\lambda} \Delta_{q r s t}, \quad \text { where } \lambda=\left\{\begin{array}{l}
1 \text { if } k \in\{q, r, s, t\} \\
0 \text { if } k \notin\{q, r, s, t\} .
\end{array}\right.
$$

Type III:

$$
\begin{gathered}
\delta_{i l}^{*}=\delta_{i l}-\alpha \delta_{i k} \text { for all } i, \quad \delta_{i j}^{*}=\delta_{i j} \text { when } i, j \neq l . \\
\Delta_{q r s t}^{*}=\Delta_{q r s t} \quad \text { if } l \notin\{q, r, s, t\} \\
\Delta_{l r s t}^{*}=\Delta_{\text {lrst }}-\alpha \Delta_{k r s t .}
\end{gathered}
$$


In particular, if $\Delta_{q r s t}=0$ always, then $\Delta_{q r s t}^{*}=0$ always.

Proof. Routine calculations, using well known relations such as $[u v, w]=[u, w][v, w]$ that hold in nilpotent groups of class 2 .

Let us say that the matrix $\bar{\Delta}$ is equivalent to $\Delta$ if $\bar{\Delta}$ is related to $\Delta$ through a finite sequence of transformations of Types I, II and III. These transformations are clearly invertible, so we are indeed dealing with an equivalence relation.

Proof of Theorem 1. The proof is by induction on $n$, the number of generators in the generating set $a_{1}, \ldots, a_{n}$ of $G$. The theorem is trivially true when $n=1$ or 2 . So take $G$ on $n$ generators, $n \geqq 3$, and assume the validity of Theorem 1 for all nilpotent groups of class 2 defined on fewer than $n$ generators. Let $c$ be an element of $G^{\prime}$ as given by (2) and such that $\Delta_{q r s t}=0$ for all $1 \leqq q, r, s, t \leqq n$. We shall prove that $c$ is a commutator. In view of the induction hypothesis we may assume that $c$ has the form

$$
c=\prod_{1 \leqq k \leqq n-1} c_{k n}^{\delta_{k n}} \prod_{1 \leqq i<j \leqq n-1} c_{i j}^{\delta_{j i}},
$$

where some $\delta_{k n}$ is non-zero. Let $d$ be the highest common factor of $\delta_{1 n}, \delta_{2 n}, \ldots, \delta_{n-1 n}$, and put $\delta_{k n}=d \alpha_{k}$ for all $1 \leqq k \leqq n-1$. Then there is a generating set $\bar{a}_{1}, \bar{a}_{2}, \ldots, \bar{a}_{n}$ of $G_{n}$ where in particular

$$
\bar{a}_{1}=a_{1}^{\alpha_{1}} a_{2}^{\alpha_{2}} \ldots a_{n-1}^{\alpha_{n}-1} \text { and } \bar{a}_{n}=a_{n}
$$

and this set is obtainable from $a_{1}, a_{2}, \ldots, a_{\mathrm{n}}$ by a sequence of elementary changes of Types I, II and III. Hence there exists a matrix $\bar{\Delta}=\left(\bar{\delta}_{i j}\right)$ equivalent to $\Delta$ and a corresponding expression for $c$ of the form

$$
c=\prod_{1 \leqq i<j \leqq n} \bar{c}_{\bar{s}_{j} j}
$$

where

$$
\begin{gathered}
\bar{\delta}_{1 n}=d \text { and } \bar{\delta}_{k n}=0 \text { for all } 2 \leqq k \leqq n-1 \text {. So } \\
\qquad c=\bar{c}_{1 n}^{d} \prod_{1 \leqq i<j \leqq n-1} \bar{c}_{i j}^{\bar{\delta}} .
\end{gathered}
$$

For the case $n=3$ this gives $c=\bar{c}_{13}^{d} \bar{c}_{12}^{\bar{b}_{12}}=\left[\bar{a}_{1}, \bar{a}_{2}^{\bar{\delta}_{12}} \bar{a}_{3}^{d}\right]$, proving that $c$ is a commutator. Suppose then that $n \geqq 4$. By Lemma $1, \bar{\Delta}_{q r s t}=0$ for all $1 \leqq q, r, s, t \leqq n$. In particular, let us choose $q, r, s$ and $t$ such that $q=1<r<s<n=t$. Since $\bar{\delta}_{k n}=0$ for $1<k<n$, we obtain $\bar{\delta}_{1 n} \delta_{r s}=0$, and hence $\bar{\delta}_{r s}=0$. Therefore

$$
c=\bar{c}_{1 n}^{d} \prod_{j=2}^{n-1} \bar{c}_{i j}^{\bar{\delta}_{j j}}
$$

which is clearly a commutator of the form $\left[a_{1}, x\right]$. This completes the proof of Theorem 1 .

3. Applications. We shall apply our test for commutators to prove the following result.

THEOREM 2. Suppose the commutator subgroup $G^{\prime}$ of a group $G$ is central in $G$ and generated by not more than two generators. Then every element of $G^{\prime}$ is a commutator. 
Proof. The case $G^{\prime}$ a cyclic group. It is sufficient to consider $G^{\prime}$ of infinite order, for it is easy to see that if $G^{\prime}$ is finite and cyclic then $G$ is a homomorphic image of a nilpotent group of class 2 with an infinite cyclic commutator subgroup. An alternative short proof is given by Macdonald [2].

Suppose then that $G^{\prime}=\langle c\rangle$, an infinite cycle. On the assumption that $G^{\prime}$ does not consist entirely of commutators, there exists a minimal positive integer $\gamma>1$ such that $c^{\gamma}$ is a commutator. Macdonald [2] showed that $G^{\prime}$ is then a two-generator group, $G^{\prime}=\left\langle c^{\gamma}, c^{\delta}\right\rangle$, where $\delta>\gamma,(\delta, \gamma)=1$ and there exist elements $a_{1}, a_{2}, a_{3}, a_{4}$ in $G$ such that

$$
c^{\gamma}=\left[a_{1}, a_{2}\right]=c_{12}, \quad c^{\delta}=\left[a_{3}, a_{4}\right]=c_{34} .
$$

Put $c_{i j}=\left[a_{i}, a_{j}\right]$ for $1 \leqq i, j \leqq 4$. Since $G^{\prime}$ is cyclic there exist relations

$$
c_{i j}=c^{\gamma_{i j}}, \quad i j=13,14,23 \text { and } 24 .
$$

Moreover, from the minimality of $\gamma$ it follows that $\gamma$ divides $\gamma_{i j}$ in each case. To see this, suppose for example that $\gamma_{13}=q \gamma+r$, where $0<r<\gamma$. Then $c^{r}=\left[a_{1}, a_{2}^{-q} a_{3}\right]$, which, being a commutator, gives a contradiction. So we may write the relations $(6)$ in the form

$$
c_{13}=c_{12}^{\alpha_{1}}, \quad c_{14}=c_{12}^{\alpha_{2}}, \quad c_{23}=c_{12}^{\alpha_{3}}, \quad c_{24}=c_{12}^{\alpha_{4}} \text {. }
$$

We now regard $G^{\prime}$ as the commutator subgroup of the subgroup $H=\left\langle a_{1}, a_{2}, a_{3}, a_{4}\right\rangle$ of $G$. Consider the change of generators of $H$ given by

$$
a_{1}^{*}=a_{1}, \quad a_{2}^{*}=a_{2}, \quad a_{3}^{*}=a_{3} a_{2}^{-\alpha_{1}}, \quad a_{4}^{*}=a_{4} a_{2}^{-\alpha_{2}} .
$$

Putting $c_{i j}^{*}=\left[a_{i}^{*}, a_{j}^{*}\right]$, we have

$$
\left\{\begin{array}{l}
c_{12}^{*}=c_{12}=c^{\gamma}, \quad c_{13}^{*}=c_{14}^{*}=1, \quad c_{23}^{*}=c_{23}=c_{12}^{* \alpha_{3}}, \\
c_{24}^{*}=c_{24}=c_{12}^{* \alpha_{4}}, \quad c_{34}^{*}=c^{\delta^{*}},
\end{array}\right.
$$

where $\delta^{*}=\delta+\gamma\left(\alpha_{2} \alpha_{3}-\alpha_{1} \alpha_{4}\right)$. Since $(\delta, \gamma)=1$ it follows that $\left(\delta^{*}, \gamma\right)=1$. Let $h$ and $k$ be integers such that $h \gamma+k \delta^{*}=1$. Therefore

$$
c=c^{h \gamma+k \delta^{*}}=c_{12}^{* h} c_{34}^{* k}\left(c_{13}^{*}\right)^{\lambda}\left(c_{24}^{*} c_{12}^{*-\alpha_{4}}\right)^{\mu},
$$

and, by virtue of relations (8), the expression (9) for $c$ is true for all integers $\lambda$ and $\mu$. To complete the proof we shall demonstrate the existence of integers $\lambda$ and $\mu$ that make the right-hand side of (9) a commutator. We note that the matrix $\Delta^{*}$ associated with the expression (9) for $c$ is such that

$$
\Delta_{1234}^{*}=\left(h-\alpha_{4} \mu\right) k-\lambda \mu .
$$

This can be made zero by choosing $\mu=1, \lambda=\left(h-\alpha_{4}\right) k$. Indeed, we observe that

$$
c=c_{12}^{* h} c_{34}^{* k} c_{13}^{*\left(h-\alpha_{4}\right) k} c_{24}^{*} c_{12}^{*-\alpha_{4}}=\left[a_{1}^{* h-\alpha_{4}} a_{4}^{*-1}, a_{2}^{*} a_{3}^{* k}\right]
$$

The case $G^{\prime}=\langle c, d\rangle$. We shall first prove that $G^{\prime}$ is generated by two commutators. Put $D=\langle d\rangle$. Then $G / D$ has cyclic commutator subgroup $\langle c D\rangle$ consisting of commutators, as has 
just been proved. So there exists an integer $\gamma$ such that $c d^{\gamma}$ is a commutator in $G$. Now put $N=\left\langle c d^{\gamma}\right\rangle$. Then $G / N$ has cyclic commutator subgroup $\langle d N\rangle$ and so there exists an integer $\mu$ such that $d\left(c d^{\nu}\right)^{\mu}$ is a commutator in $G$. The commutators $c d, d\left(c d^{\nu}\right)^{\mu}$ generate $G^{\prime}$.

Let us simplify the notation and take $c$ and $d$ to be commutators generating $G^{\prime}$. We put

$$
c \doteq\left[a_{1}, a_{2}\right]=c_{12}, \quad d=\left[a_{3}, a_{4}\right]=c_{34},
$$

and proceed to show that every element of $G^{\prime}$ is a commutator in the subgroup $H$ of $G$ generated by $a_{1}, a_{2}, a_{3}, a_{4}$. As usual, put $c_{i j}=\left[a_{i}, a_{j}\right]$. There exist relations

$$
c_{13}=c_{12}^{\alpha_{1}} c_{34}^{\beta_{1}}, \quad c_{14}=c_{12}^{\alpha_{2}} c_{34}^{\beta_{2}}, \quad c_{23}=c_{12}^{\alpha_{3}} c_{34}^{\beta_{3}}, \quad c_{24}=c_{12}^{\alpha_{4}} c_{34}^{\beta_{4}} \text {. }
$$

Suppose that not all $\beta_{i}$ are zero. Suppose also that of all presentations of $H$ by 4 generators subject to relations of type (10) our presentation involves the numerically smallest possible nonzero index of $c_{34}$. By a relabelling of generators, if necessary, we can ensure that this index is $\beta_{1}$.

We claim that $\beta_{1}$ divides $\beta_{2}$. For if not, there exist $q$ and $r$ such that $\beta_{2}=q \beta_{1}+r$, $0<r<\left|\beta_{1}\right|$. The Type III transformation $a_{4}^{*}=a_{4} a_{3}^{-q}, a_{i}^{*}=a_{i}, i=1,2,3$, yields relations of type (10) and including $c_{14}^{*}=c_{12}^{*}{ }^{\alpha_{2}} c_{34}^{* r}$, and this contradicts the minimality of $\beta_{1}$. By performing a Type III transformation just described we may arrange that $\beta_{2}=0$. Similar considerations apply to $\beta_{3}$, which may be set to zero without affecting the values of $\beta_{1}$ or $\beta_{2}$.

Thus the relations (10) may be taken to be of the form

$$
c_{13}=c_{12}^{\alpha_{1}} c_{34}^{\beta_{1}}, \quad c_{14}=c_{12}^{\alpha_{2}}, \quad c_{23}=c_{12}^{\alpha_{3}}, \quad c_{24}=c_{12}^{\alpha_{4}} c_{34}^{\beta_{4}} .
$$

This form also applies (with $\beta_{1}=\beta_{4}=0$ ) to the case that in (10) all $\beta_{i}$ are zero.

Now let $c$ be an arbitrary element of $G^{\prime}$. Then there exist integers $\delta, \varepsilon$ such that, for all integers $\lambda$ and $\mu$,

$$
c=c_{12}^{\delta} c_{34}^{c}\left(c_{14} c_{12}^{-\alpha_{2}}\right)^{\lambda}\left(c_{23} c_{12}^{-\alpha_{3}}\right)^{\mu} .
$$

Let $\Delta$ be the $4 \times 4$ matrix associated with the expression (12) for $c$. Then

$$
\Delta_{1234}=\left(\delta-\alpha_{2} \lambda-\alpha_{3} \mu\right) \varepsilon+\lambda \mu=\mu\left(\lambda-\alpha_{3} \varepsilon\right)-\varepsilon\left(\alpha_{2} \lambda-\delta\right),
$$

and this can be made zero by choosing

$$
\lambda=\alpha_{3} \varepsilon+1, \quad \mu=\varepsilon\left(\alpha_{2} \hat{\lambda}-\delta\right) .
$$

Therefore the element $c$ of $G^{\prime}$ is a commutator. (Using the above values it will be found that

$$
c=\left[a_{1}^{\alpha_{3} \varepsilon+1} a_{3}^{\varepsilon}, a_{4} a_{2}^{\delta-\alpha_{2}-\alpha_{2} \alpha_{3} \beta}\right],
$$

an expression which would hardly have been discovered without an algorithmic approach.) This completes the proof of Theorem 2.

Concerning generalizations of Theorem 2, we do not know whether the result extends to rank 3 central commutator subgroups. In this direction Rodney [3] has shown that a finite commutator subgroup that is central and is generated by not more than 3 generators contains commutators only. Finally, Theorem 2 does not extend to rank 4 commutator subgroups. Consider the quotient group

$$
G_{4} / R, \text { where } R=\left\langle c_{13}, c_{14}\right\rangle \text {. }
$$


The commutator subgroup $\left(G_{4} / R\right)^{\prime}$ is torsion free and of rank 4. The element $c_{12} c_{34} R$ is not a commutator, for no element $c_{12} c_{34} c_{13}^{\lambda} c_{14}^{\mu}$ of $G_{4}^{\prime}$ is a commutator. This follows from Macdonald's argument.

I should like to acknowledge with thanks some stimulating discussions on the subject of this note with David Rodney, at a time when he was my research student. He is presently at the University of Ibadan.

\section{REFERENCES}

1. I. D. Macdonald, On a set of normal subgroups, Proc. Glasgow Math. Soc. 5 (1962), 137-146.

2. I. D. Macdonald, On cyclic commutator subgroups, J. London Math. Soc. 38 (1963), 419-422.

3. D. M. Rodney, Commutators and conjugacy in groups, Ph.D. thesis, University of Keele (1974).

THE UNIVERSITY

Keele

STAFFS. ST5 5BG 